\title{
THE MANX MUON COOLING DEMONSTRATION EXPERIMENT *
}

\author{
K. Yonehara\#, V.I. Balbekov, D. Broemmelsiek, M. Hu, A. Jansson, Vadim Kashikhin,
}

Vladimir Kashikhin, M. Lamm, N. V. Mokhov, V. Shiltsev, V. Yarba, Fermilab, Batavia, IL R. J. Abrams, M. A. Cummings, R. P. Johnson, S. A. Kahn, T. J. Roberts, Muons, Inc., Batavia, IL

\section{Abstract}

MANX is an experiment to prove that effective sixdimensional (6D) muon beam cooling can be achieved in a Helical Cooling Channel (HCC) using ionizationcooling with helical and solenoidal magnets in a novel configuration. The aim is to demonstrate that $6 \mathrm{D}$ muon beam cooling is understood well enough to plan intense neutrino factories and high-luminosity muon colliders. The experiment consists of the HCC magnet that envelops a liquid helium energy absorber, upstream and downstream instrumentation to measure the beam parameters before and after cooling, and emittance matching sections between the detectors and the HCC.

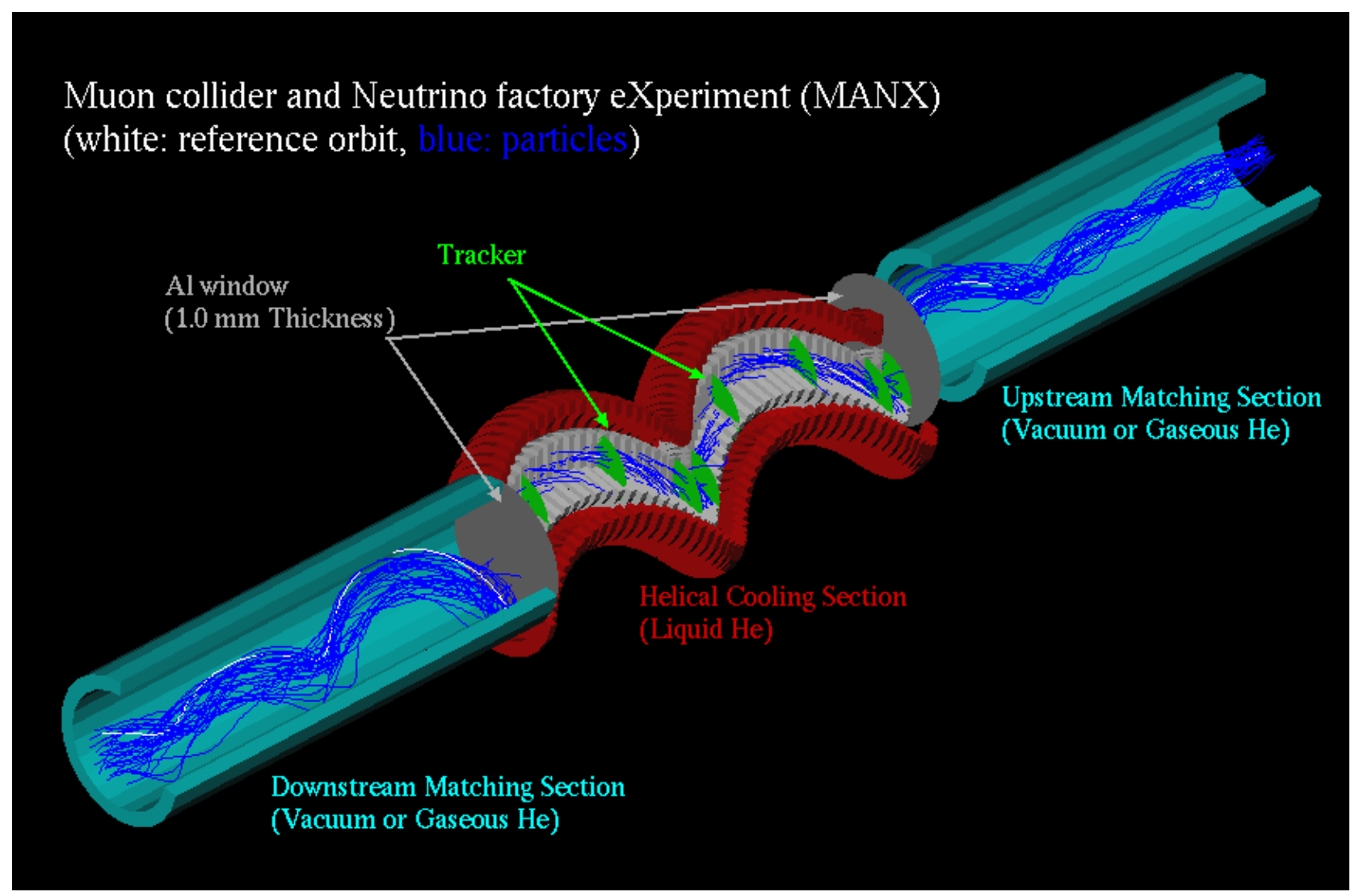

Figure 1: Conceptual picture of the helical cooling channel (red) and the two emittance matching sections (blue). The helical solenoid magnets shown in red enclose the LHe ionization energy absorber, which is separated from the vacuum of the matching sections by thin Al windows. The beam is physically larger after cooling because it has much less momentum than the incoming beam; the normalized emittance has been reduced. The total length is 9.6 meters.

\section{INTRODUCTION}

A muon collider at the energy frontier can be used to explore physics beyond the standard model such as supersymmetry, technicolor, extra dimensions, and grand unified theories [1]. A muon collider at the Higgs mass can profit from the s-channel resonance production cross section that is 40,000 times more than an electron collider to allow the fundamental properties of the Higgs boson to be measured more precisely. A muon beam which is produced via pion decay needs fast $6 \mathrm{D}$ phase space

*Supported DOE STTR grant DE-FG02-06ER86282 \#yonehara@fnal.gov emittance reduction to fit into the acceptance of the RF cavities, for instance, being developed for the ILC. The ionization cooling method is the only way to shrink muon beam transverse phase space within its short lifetime (2.2 $\mu$ sec in its rest frame) [2]. However, emittance exchange is required to provide longitudinal phase space cooling.

Recently, a novel cooling scheme has been proposed [3]. A HCC is used with a continuous ionization cooling absorber. The helical magnet produces helical dipole, helical quadrupole, and solenoidal field components. The muon beam in the magnet has a spiral orbit with a constant helical orbit radius and a constant helical period. The spiral orbit is generated by a repulsive radial force 
which is induced by the muon's longitudinal momentum with the transverse helical dipole field component and an attractive radial force which is induced by the muon's transverse momentum with the solenoidal field component. Each muon oscillates about an equilibrium orbit with an amplitude that depends on the momentum. A particle with a higher (lower) momentum than the equilibrium particle has a longer (shorter) path length, and loses more (less) kinetic energy in the continuous energy absorber. In this way emittance is exchanged from the longitudinal to the transverse phase space. For a system of multiple cooling channels, the lost kinetic energy is restored by RF cavities between channels or integrated RF cavities. The analytical treatment of this cooling scheme has shown a 6D cooling factor to be of order $10^{6}[3]$.

Simulations have verified the helical cooling theory [4]. For the next stage, we are planning a demonstration experiment called MANX (Muon collider And Neutrino factory eXperiment) [5]. In this paper, we discuss the concepts of the MANX experiment and describe the recent progress on designing the matching sections.

\section{CONCEPTUAL DESIGN OF MANX CHANNEL}

Figure 1 shows the conceptual picture of the MANX channel. It consists of a helical cooling channel (red) and the upstream and downstream matching section magnets (light blue). The liquid helium (LHe) ionization cooling absorber fills the cooling channel. The beam in the matching sections is assumed to be in vacuum in Figure 1. There are thin $\mathrm{Al}$ windows between cooling and matching magnets to separate liquid and vacuum regions.

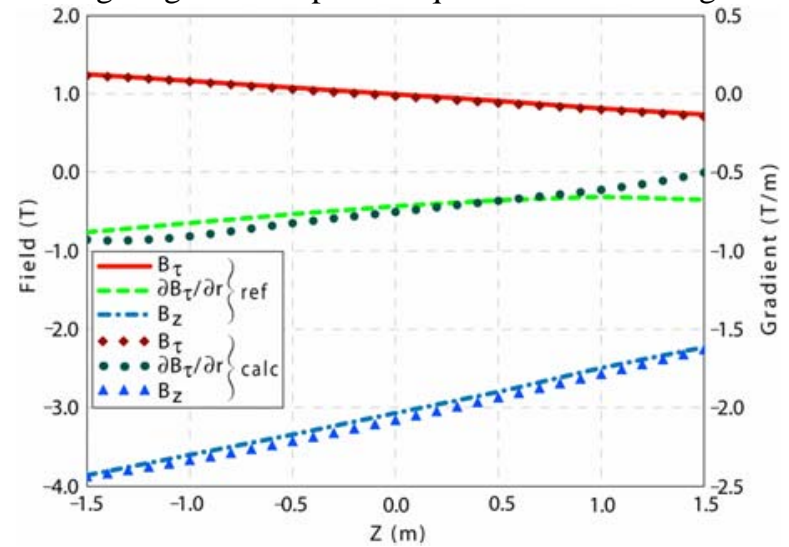

Figure 2: Field configurations along the design orbit in the cooling channel. The circle, square, and triangle points show the required analytical field components and gradients and red, green, and blue lines are calculated by finite element analysis (FEA). The conductor design is shown in Ref. [6].

Emittance exchange can take place without an RF cavity. Therefore, for simplicity, there are no RF cavities in the current MANX design. Hence the magnetic field strength in the cooling channel is varied to correspond to the momentum decrease along the channel. Figure 2 shows the designed field strength and gradients as a function of the length of channel. In the current design, the initial and final mean momenta of the muons are 300 $\mathrm{MeV} / \mathrm{c}$ and $170 \mathrm{MeV} / \mathrm{c}$ for a channel length of $3.2 \mathrm{~m}$. LHe is chosen as the ionization cooling absorber material in the current design because of safety issues and its possible use as a coolant for the magnet conductors. Table 1 has a summary of the design parameters of MANX channel.

\begin{tabular}{|c|c|c|}
\hline $\begin{array}{l}\text { Initial mean momentum: } \\
\text { Final meam momentum }\end{array}$ & $\mathbf{P}$ & $\begin{array}{l}300 \mathrm{MeV} / \mathrm{c} \\
170 \mathrm{MeV} / \mathrm{c}\end{array}$ \\
\hline Helical pitch: & $\kappa$ & 1 \\
\hline Helical period: & $\lambda$ & $1.6 \mathrm{~m}$ \\
\hline Helical ref. orbit radius: & $a$ & $0.255 \mathrm{~m}$ \\
\hline $\begin{array}{l}\text { Initial solenoid strength: } \\
\text { Final solenoid strength: }\end{array}$ & $\mathbf{B z}$ & $\begin{array}{l}-3.8 \mathrm{~T} \\
-1.7 \mathrm{~T}\end{array}$ \\
\hline $\begin{array}{l}\text { Initial helical dipole strength: } \\
\text { Final helical dipole strength: }\end{array}$ & b & $\begin{array}{l}1.2 \mathrm{~T} \\
0.8 \mathrm{~T}\end{array}$ \\
\hline $\begin{array}{l}\text { Initial helical quad. strength: } \\
\text { Final helical quad. Strength: }\end{array}$ & b' & $\begin{array}{l}-0.9 \mathrm{~T} / \mathrm{m} \\
-0.5 \mathrm{~T} / \mathrm{m}\end{array}$ \\
\hline
\end{tabular}

Table 1: Current MANX design parameters. Helical Pitch, $\kappa$, is defined as the tangent of the helical pitch angle of the reference orbit. Helical field strengths are quoted at the radius of the helical reference orbit, $a$.

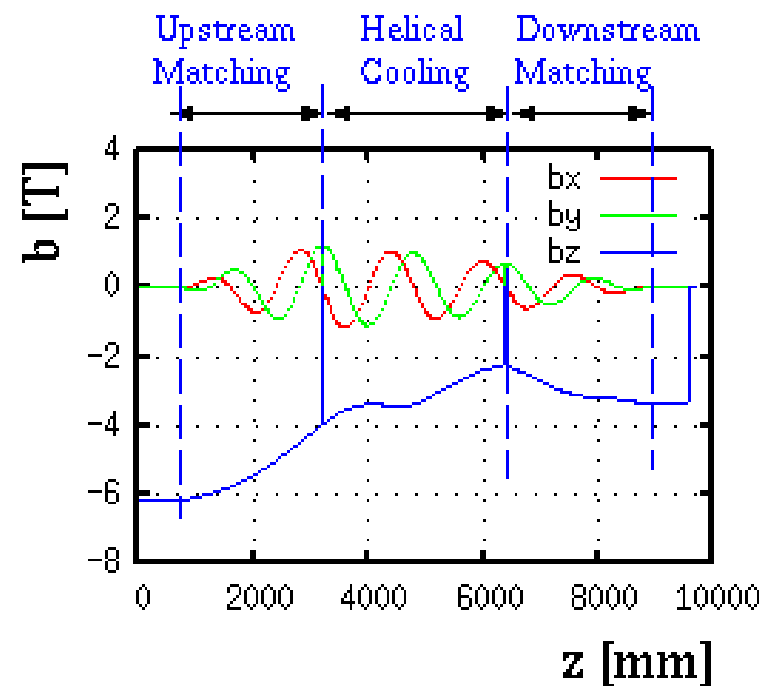

Figure 3: Field strength along the reference orbit in the MANX channel.

The function of the matching magnets is to match the emittance of the injected beam into the acceptance of the helical magnet. To do this, it must induce a transverse momentum kick to match the helical pitch ( $\kappa)$ and make a beam position offset to match the helical orbit radius $(a)$. The first attempt adiabatically ramped the helical dipole and quadrupole components while holding the solenoid component constant. The required matching length was more than $10 \mathrm{~m}$, but it makes a perfect match. The period of the betatron oscillation induced by the ramping function is found to be $1.5 \lambda$. Hence, it was decided that 
the transverse momentum kick should take place during one betatron oscillation, while the beam stability is maintained by tuning the solenoid field strength. Figure 3 shows the magnetic field configuration in the $3.2 \mathrm{~m}$ cooling channel and $2.4 \mathrm{~m}$ matching sections.

As shown in Figure 4, there is emittance growth in the matching sections, which indicates there is a mismatching in the matching sections. This is caused by the beta beat. We need to improve the design of the matching magnets to make a practical matching magnet.

The field parameters are slightly different from the design values because of the imperfection of the beta beat tuning in the matching magnets. The average beam position at the end of the cooling channel is $\sim 10 \mathrm{~mm}$ in $\mathrm{x}$ and y directions.

\section{SIMULATION RESULT}

The program G4Beamline [7] was used to study the cooling behavior of the MANX experiment. Figure 1 is an example of its visual utility. Figure 4 shows the evolution of the normalized rms 6D emittance down the length of the channel. The cooling factors in the longitudinal and in each transverse direction are equally 1.3, hence the cooling factor of $6 \mathrm{D}$ emittance is approximately 2.1 .

The simulated injected beam is large to probe the acceptance of the cooling channel. The particles used for Figure 4 are those that pass through the whole cooling channel. Hence, it includes particles traversing barely stable regions of the magnet aperture. As a result, we observe $6 \mathrm{D}$ emittance growth in the matching magnets.

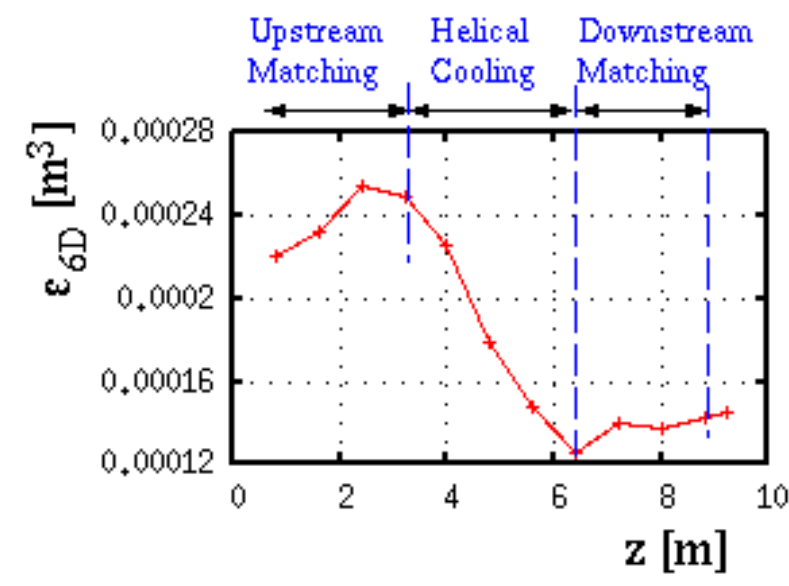

Figure 4: 6D emittance evolution in MANX.

\section{EXPERIMENTAL DESIGN CONCEPTS}

We are investigating several designs for the MANX experiment. One design is similar to the MICE experiment, in which the emittances are reconstructed from ensembles of individual beam particles. Measurement of the longitudinal component of the momenta permits reconstruction of the longitudinal emittance cooling, which is essential for the proof of the emittance exchange. To determine the momentum reduction and evolution of time structure measurements through the cooling channel we are considering installing scintillating fiber detectors inside of the cooling section and the matching sections, which will be accommodated in the design of the magnets. Particle identification detectors, such as time-of-flight and Cherenkov counters are envisioned, and a range measurement downstream of the system can be used to provide additional verification of muon identity and final energy. Another design utilizes external spectrometers to measure the initial and final momenta.

Another approach, discussed in Ref. [8], called the beamlet method, uses highly collimated, small diameter beamlets to probe the behavior of the beamlets as they pass through the cooling channel. To cover the acceptance of the channel it is necessary to make a series of measurements to cover the acceptance of the channel. In this approach, with sufficient beam intensity it is possible to operate scintillating fibers in a beam profiling mode rather than in a single particle trajectory mode.

\section{Muon beam line/transport line}

Designs of the pion production target, the pion decay channel, and the beam transport system in the Fermilab MuCool Test Area are in progress [8]. We are also investigating a number of other beam lines and experimental areas at Fermilab and other laboratories that could be suitable for the MANX experiment.

\section{CONCLUSIONS}

A conceptual design study of a MANX channel consisting of a $3.2 \mathrm{~m}$ helical cooling magnet with $2.4 \mathrm{~m}$ matching magnets is presented. The matching magnet successfully produces the required transverse momentum and the position off-set from the coaxial beam. However, it does not result in a perfect match to the beam phase space. We need to improve the matching magnet design. The cooling channel reduces the $6 \mathrm{D}$ emittance by a factor of 2 .

Two different approaches to measuring cooling are discussed; the single particle tracking and the beamlet methods. Possible instrumentation to measure the beam emittance is discussed.

\section{REFERENCES}

[1] The applications of the low emittance muon collider were discussed in the theory part in the LEMC07 workshop, http://www.muonsinc.com/mcwfeb07/

[2] D. Neuffer, Part. Accel. 14, pp.75 (1983)

[3] Y. Derbenev and R. P. Johnson, PRSTAB 8,041002 (2005)

[4] K. Yonehara et al., PAC05, TPPP052

[5] K. Yonehara et al., COOL05, P23

[6] V. Kashikhin et al., PAC07, 2145-MOPAS012

[7] T. J. Roberts and D. M. Kaplan PAC07, 2141 - THPAN103

[8] A. Jansson et al., PAC07, 3027-THPMN102 